\title{
CHANGING ADHESION FORCE FOR TESTING ROAD VEHICLES AT SAFE SPEED
}

\author{
Petr Jilek, Jan Nemec \\ University of Pardubice, Czech Republic \\ petr.jilek@upce.cz,nemec.jan@upce.cz
}

\begin{abstract}
The article deals with various ways of bringing a car to the stability limit in terms of adhesion conditions. The article deals with the possibilities how to reduce the adhesion force transmitted between the car wheels and the road. Subsequently, we evaluate the change in the behaviour of the car depending on the way the adhesion force changes. The measurement is carried out on a special experimental vehicle. The aim of the article is to find the differences in the car behaviour when inducing a limit situation. In the first case, by reducing the coefficient of adhesion and, in the second case, by reducing the radial response on the car wheels. The change of the coefficient of adhesion is done by means of a sliding surface (foil) sprayed with water. To reduce the radial response on vehicle wheels, we used a device called Alternative SkidCar. It is similar to a commercially available SkidCar device that is built for a particular experimental vehicle. The car behaviour in both cases was verified on the basis of driving tests in a direct direction. The actual test was carried out in the form of critical braking. Initial set of measurements was realized for dry road and separate vehicle. Subsequently, we proceeded to the experiment on the sliding surface. The coefficient of adhesion is reduced to a constant value on the sliding surface. The last set of experimental tests was performed with the help of Alternative SkidCar. We carried out the initial measurements for a situation where the car wheels transmitted a full radial response. In this way we found out to what extent the frame of the Alternative SkidCar influences the car behaviour. Furthermore, we reduced the radial response of vehicle wheels in three stages. Subsequently, we processed the measured values and, by comparison, we found differences from comparable tests. Due to the reduced adhesion force, a limit state in relation to stability at lower speeds can be induced on the vehicle. In this way, it is possible to achieve skid at a lower, so-called safe speed.
\end{abstract}

Keywords: road vehicle, adhesion force, stability limit, critical braking, radial reactions.

\section{Introduction}

The main element capable of affecting the stability of the vehicle is the adhesive force that is transmitted between vehicle wheels and the road. It is this force that determines the maximum forward or braking deceleration a vehicle can achieve, thus limiting its dynamics. At the same time, the forward movement should be evaluated taking into account the lateral forces. If the driving situation requires more force to be transmitted than the adhesive force, the balance of forces is disturbed and the vehicle skids. The actual form of skid is given by the vehicle structure, tyres used and the position of the vehicle's centre of gravity. The safety of road vehicles in motion is mainly due to their stability. Vehicles are tested particularly for longitudinal and transverse stability and rollover threshold. Ideally, vehicles are tested under real conditions. However, it is not always possible. For example, from the point of view of natural conditions or in the field of verification of vehicle prototypes. It is precisely in order to reduce the possibility of revealing a newly prepared vehicle by competitors, car manufacturers approach to testing under secret conditions. It is in this area of development that artificial (model) conditions [1-3] are very often used. The frictional properties of a vehicle tire usually depend on the road surface. It is influenced by various natural conditions, the macro-profile of the road surface, foreign particles, such as dust or snow [4]. Stability depends on the type of road and its roughness and geometrical parameters [5]. The analysis of driving characteristics with vehicle stability is described by road data, lateral and longitudinal acceleration, vehicle body yaw rate, wheel angular speed, steering wheel angle [6] and body roll and inclination. In terms of driving stability of a car, it is possible to test cars under conditions where the adhesive force is reduced by reducing the coefficient of adhesion or by reducing the radial reaction transmitted by the vehicle wheels to the road. In the first case, sliding surfaces or sliding tyres are used, for example with the designation Easy Drift Ring [7]. Reducing the radial response of vehicle wheels is possible by using commercially available SkidCar [6] or SlideWheel [8]. The advantage of the above methods is a verification of car behaviour when driving at a safe speed. The use of model conditions brings a different degree of simplification, where the test drives may vary slightly according to the conditions used [9]. The only category for evaluating the stability of a car is the simulation of the tire-road contact using finite elements $[1 ; 10]$.

The paper tries to mutually influence the behavior of cars with reduced adhesive force. We compare the behavior of a car with reduced adhesion force due to a decrease in the coefficient of 
adhesion with a reduced adhesion force based on a decrease in the radial reaction of the vehicle wheel. In the first case, the course of the car's behavior on the slippery surface is measured. The coefficient of adhesion is defined by the properties of the sliding surface and is constant. In order to reduce the coefficient of adhesion and to prevent wear of the sliding surface, the surface is sprinkled with water. The second course for comparison is the course of an experimental car with the Alternative SkidCar (ASC) system. In this way, it is possible to reduce the adhesive force according to the current need, even during the implementation of the experiment. The advantage of ASC is the zero treatment of the test surface, the disadvantage is the increase in the weight of the car and the reduction of tilting of the vehicle body.

\section{Materials and methods}

Regarding the mutual comparison, we achieved the goal in two ways. The first method is by reducing the adhesive force using a substrate with a reduced coefficient of adhesion (Fig. 1). As a second way to reduce the radial response of vehicle wheel, we used the Alternative SkidCar (ASC) (Fig. 2). It is similar to commercially available SkidCar adapted for an experimental vehicle. An experimental vehicle was used for test drives, which was specifically designed to verify driving stability. For experimental measurements, there were no electronic systems active on the vehicle that would affect the stability of the vehicle. The limit state in which the stability limit is exceeded, is the forward skid of the vehicle.

ASC is a device that is retrofitted to the experimental vehicle. The main part is a steel frame that is attached to the vehicle. ASC support wheels are attached to the sides of the frame. By changing the position of these wheels, the radial response transmitted by the vehicle wheels is reduced and the amount of adhesive force is changed. The difference between ASC and the commercially available SkidCar is the change in the radial response on vehicle wheels, which is done mechanically.

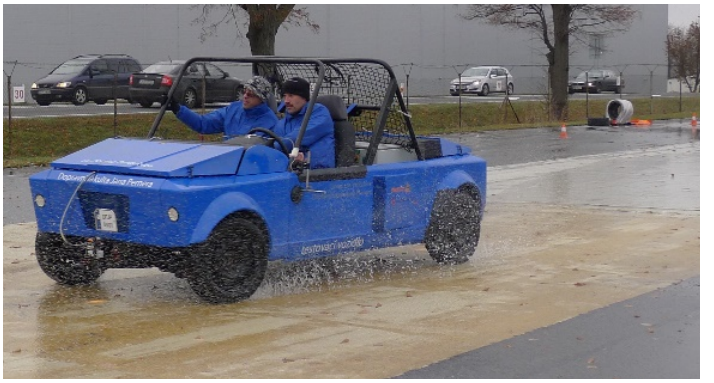

Fig. 1. Experimental vehicle on sliding surface

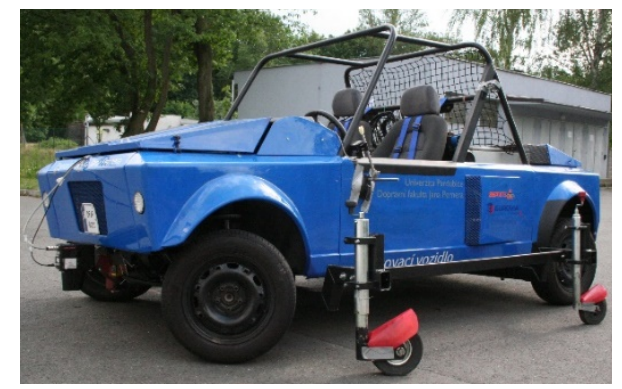

Fig. 2. Experimental vehicle with ASC

We chose panic braking in a straight line as suitable test drives. It means braking with maximum force on the brake pedal. Due to the fact that no driver assistant is intentionally active on the car, the vehicle wheels are locked. Test drives were carried out with a driver and a passenger in the car. Test corridor was outlined with cones according to Fig. 3. We increased the corridor width for the Alternative SkidCar to $t_{4}^{\prime}$. The width is given by the width of the initial corridor $t_{4}$ to which twice $300 \mathrm{~mm}$ is added, which is the overlap of the ASC frame over the vehicle plan.

The aim of the chosen test drive is to compare the difference in the behaviour of the experimental vehicle in direct driving during intensive braking. Since the test drives are carried out without a steering automation (the car is driven by a man), it is not possible to provide the same high control force on the brake pedal for all measurements. That is why we deliberately chose braking with the maximum control force on the brake pedal that causes forward skidding.

The start of braking in the data record is determined by the signal from the brake light sensor. We applied the control force on the brake pedal in a step manner from zero to the maximum, so-called 'panic' braking. The default speed for the braking mode was selected from an interval of $30 \mathrm{~km}^{\prime} \mathrm{h}^{-1}$ $\left( \pm 5 \mathrm{~km}^{-1}\right)$. Before entering the measurement corridor, it is necessary to hold the vehicle for at least $1 \mathrm{~s}$ without traction and braking (it is necessary to disengage the clutch) and then brake with maximum possible control force on the brake pedal.

A series of verification measurements must be performed before starting a measurement or after re-setting alternative SkidCar. Verification measurements are used to verify the functionality of the 
measurement chain and as a data set for subsequent off-time correction. The correction is necessary in order to eliminate mounting and technical deviations when installing the measuring apparatus on the vehicle. At the same time, it is essential to monitor the initial position of the vehicle body when changing the ASC settings to simulate the desired adhesion conditions. Verification measurements of all quantities were carried out in the form of repeated records in the range of seven measurements with steady driving in a straight direction on a $30 \mathrm{~m}$ track and in both directions. The results of the verification measurement are quasi-static waveforms of measured quantities for direct driving on horizontal road (marked with index 0). We removed outliers that were not related to the vehicle's behaviour from the compensation measurement waveforms. Subsequently, we performed the smoothing of measured waveforms using averaging. The result is longitudinal $a_{x 0}$ and transverse $a_{y 0}$ acceleration waveforms, height coordinates of the front $H_{1 o}$ and rear $H_{2 o}$ of the body, directional deviation $\varphi_{0}$, yaw rate $\omega_{0}$ and steering wheel turning angle $\beta_{v o}$, which were used for off-time correction of the waveforms from the measured test drives.

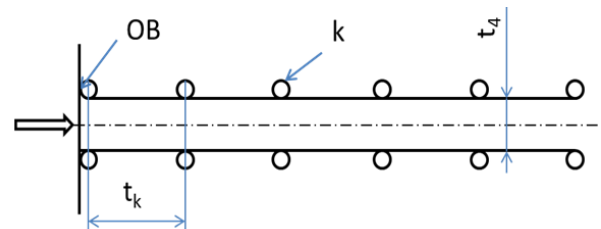

Fig. 3. Test corridor sliding surface

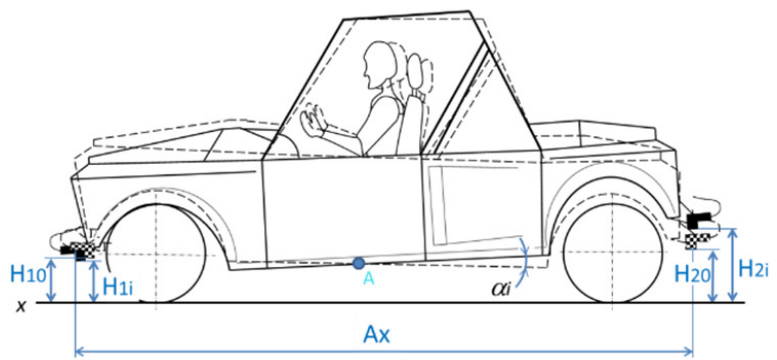

Fig. 4. Plane model of body tilt

The first series of test drives was carried out by the experimental vehicle on a sliding surface. In order to verify the behaviour of the vehicle in a state of unreduced adhesion conditions, we performed the first series of measurements on dry asphalt. The coefficient of adhesion by experimental measurement was $\varphi=0.9$ in this case [11]. Subsequently, we repeated the experimental measurements for the sliding surface. Using the experimental measurement, we found the coefficient of adhesion of sliding surface of $\varphi=0.4$.The second series of test drives was carried out by the experimental vehicle supplemented with the Alternative SkidCar system. Again, we performed the first series of measurements for dry asphalt. Subsequently, we performed the measurements for a $50 \%$ reduction in the radial response of vehicle wheels.During the test drives we measured physical quantities that clearly described the behaviour of the car, namely vehicle speed $v$, steering wheel turning angle $\beta_{v}$, vehicle yaw rate $\omega$, vehicle yaw angle $\Psi$, vehicle yaw rate $\omega$, yaw angle $\varphi$, longitudinal vehicle acceleration $a_{x}$, transverse vehicle acceleration $a_{y}$, start of braking $B S$ and body height from road $H_{1}$ and $H_{2}$. Due to the limited scope of the paper, only the main measured waveforms are presented as diagrams. The change in the body tilt position $\alpha_{i}$ is shown in Fig. 4 and, taking into account the compensatory measurement, we determine it according to relation (1) from the measured values of the ultrasonic height sensors.

$$
\alpha_{i}=\tan ^{-1}\left(\frac{\left(H_{1 i}-H_{1 o}\right)-\left(H_{2 i}-H_{2 o}\right)}{A_{x}}\right),
$$

where: $\alpha_{i}$ - body tilt angle, ${ }^{\circ}$;

$H_{10}$ - vertical distance from the measuring point to the road at the front of the vehicle corresponding to the compensation measurement, $\mathrm{mm}$;

$H_{20}$ - vertical distance of the measuring point from the road at the rear of the vehicle corresponding to the compensation measurement, $\mathrm{mm}$;

$A_{x}$ - distance between the height sensors in the longitudinal plane of the vehicle, mm;

$H_{1 i}$ - vertical distance of the meas. point from the road at the front of the body at $t_{i}, \mathrm{~mm}$;

$H_{2 i}$-vertical distance of the meas. point from the road at the rear of the body at $t_{i}, \mathrm{~mm}$.

A wire sensor was used to measure the steering wheel turning angle. It is a resistive analog distance sensor, where the current position is given by pulling the wire from the sensor body. As we needed to know the steering wheel turning angle, we let the wire wind up on the steering wheel shaft. 
We recalculated the current value of the extension according to formula (2) to the steering wheel turning angle.

$$
\beta_{v}=\frac{180 \cdot l_{l}}{\pi \cdot r_{v}}-\beta_{v o},
$$

where: $\beta_{v}$ - steering wheel turning angle, ${ }^{\circ}$;

$r_{v} \quad$ - steering column shaft radius, $\mathrm{mm}$;

$l_{l} \quad$ - wire extension from steering wheel turning angle sensor, $\mathrm{mm}$;

$\beta_{v o}$ - steering wheel turning angle in verification measurement, ${ }^{\circ}$.

To ensure repeatability, we repeated each test drive ten times. After comparing and verifying the validity of measurements, we removed the outliers. If more than 2 tests were obviously different, we identified the cause and repeated the whole set of test drives.We smoothed the signals from the distance sensors, yaw angle and vehicle yaw rate using moving average at the interval $t=0.2 \mathrm{~s}$. Subsequently, we used off-time correction according to the data obtained during the compensation measurement.

\section{Results and discussion}

Graphical waveforms are presented in the form when at time $t=1 \mathrm{~s}$ the signal $B S$ changes from zero to 0.8 . This is when the driver comes to brake. Fig. 5-9 show the left waveform always corresponding to the mutual comparison of driving on dry asphalt, the right waveform is a comparison of the sliding surface with $50 \%$ radial reaction of vehicle wheels in ASC.
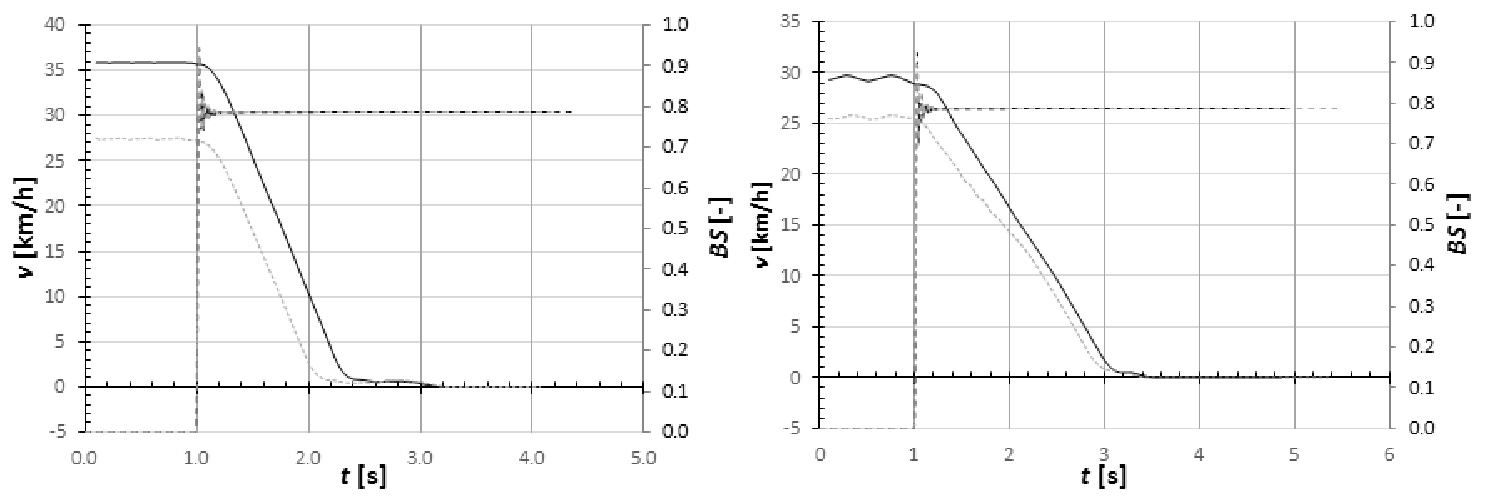

dry asphalt sliding surface $+50 \%$ ASC

Fig. 5. Vehicle speed $v$ : (- ASC, --- sliding surface)
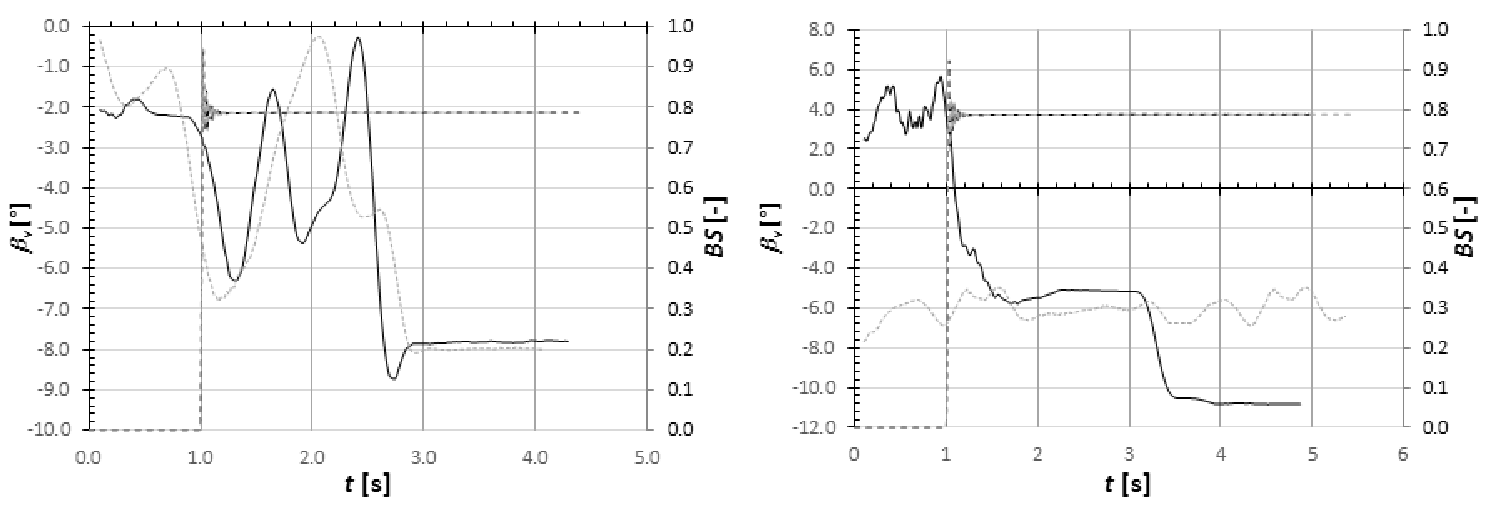

dry asphalt sliding surface $+50 \%$ ASC

Fig. 6. Steering wheel turning angle $\beta_{v}$

Difference in initial speed when entering the test corridor (Fig. 5) is caused by the driver. The driver had a speedometer located outside his visual field, so the initial speed is given by his subjective perception. It was not possible to fix the steering wheel to ensure safety during experimental measurements. Therefore, the position of the steering wheel for direct driving is determined by the 
driver's skills. Significant steering wheel turning angle (Fig. 6) occurs only when the vehicle has stopped. Therefore, intervention in the steering wheel does not bring an error into the measurement.
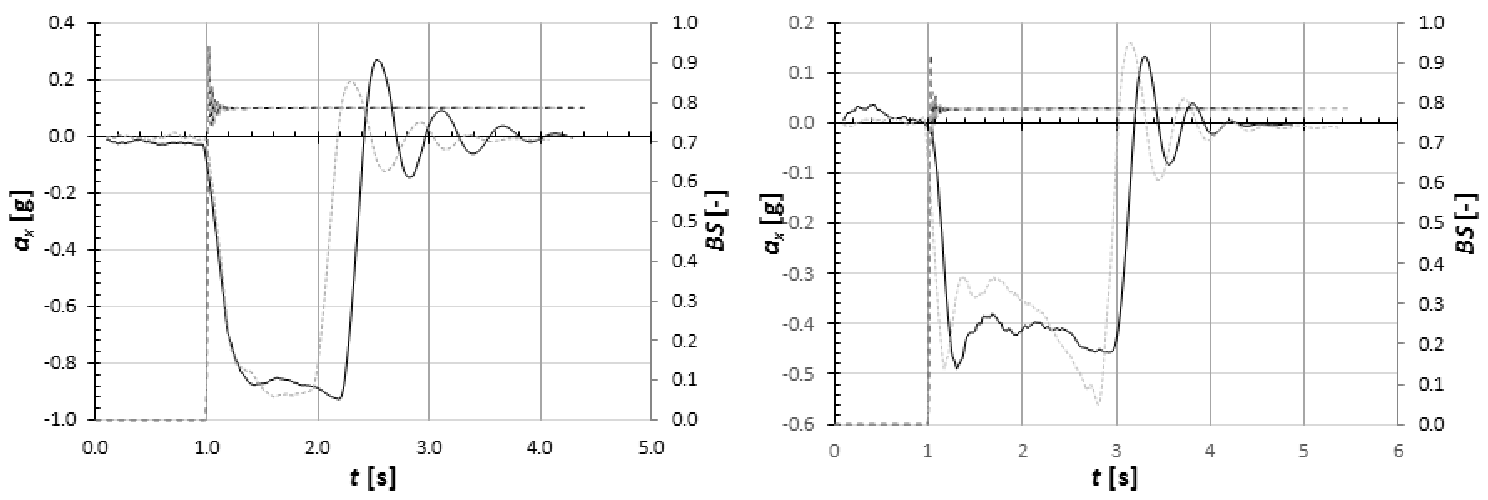

dry asphalt $\quad$ sliding surface $+50 \%$ ASC

Fig. 7. Longitudinal acceleration $a_{x}$
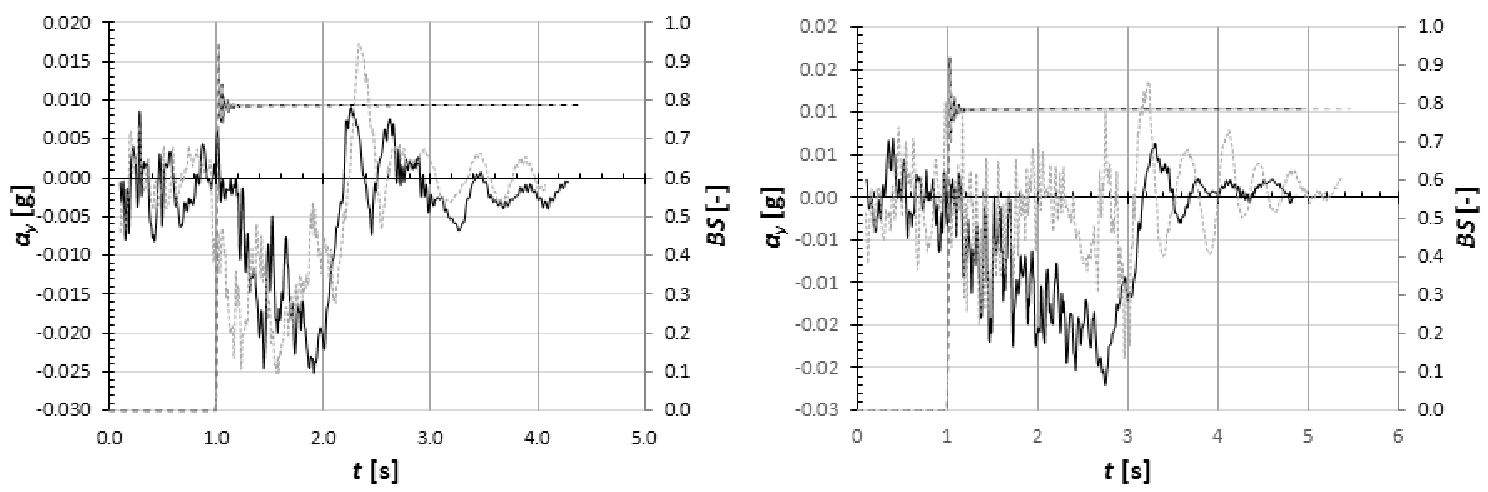

dry asphalt

sliding surface $+50 \%$ ASC

Fig. 8. Transverse acceleration $a_{y}$
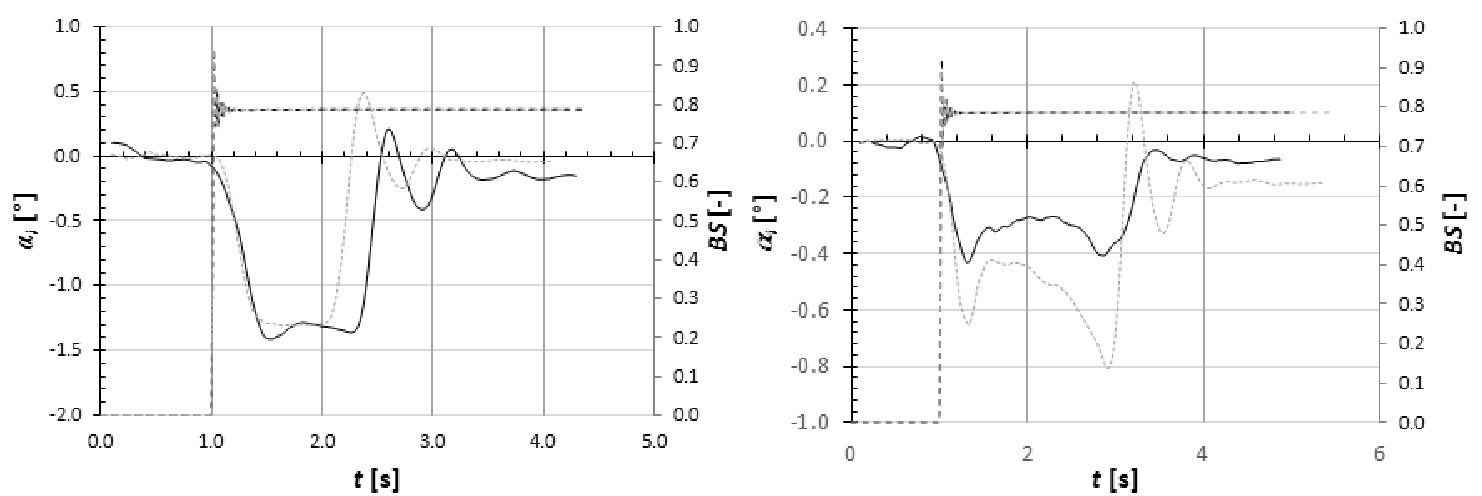

dry asphalt

sliding surface $+50 \%$ ASC

Fig. 9. Body tilt angle $\alpha_{i}$

When braking at skid level in a straight line, longitudinal deceleration $a_{x}$ takes place at axis $x_{0}$. At the same time, the forward velocity $v_{x}$ is reduced and the fictitious force $F_{i}$ acting on the centre of gravity of the vehicle parallel to the longitudinal axis of the vehicle is generated. The amount of the adhesive force $F_{a d}$ is dependent on the coefficient of adhesion, as well as on the size of slide with which the car wheels roll, therefore the maximum value of the longitudinal deceleration and thus the adhesive force is at the beginning of braking. Due to the increase in slide of the wheel relative to the road, the adhesive force decreases slightly and with it the longitudinal deceleration. The decrease in longitudinal deceleration is apparent from the measured waveforms in Fig. 7. When the vehicle is stationary, an overshoot occurs during the measured waveform of the forward acceleration caused by the body swinging when the vehicle stops. Due to the same road adhesion properties under the left and 
right vehicle wheels and asymmetric brakes up to $5 \%$, the amount of transverse acceleration is negligible Fig. 8 and corresponds to direct driving.

The fictitious force acting on the centre of gravity of the vehicle causes the body tilt $\alpha_{i}$. As a result, the front wheels of the car are loaded and, due to a higher radial response, are able to transmit a higher adhesive force than the rear wheels. It is apparent from Fig. 9 that the vehicle body tilt is comparable to the vehicle without ASC when driving the vehicle with ASC with full radial response on vehicle wheels (ASC wheels carry zero load). The difference is when the vehicle wheels transmit a $50 \%$ radial response. Due to the design and arrangement of ASC, the body tilt is determined by the stiffness of the support wheels and their location in the longitudinal axis of the vehicle. From this point of view, it is apparent that the body tilt in ASC is lower than that in the experimental vehicle braking on a sliding surface.

\section{Conclusions}

With a constant value of the coefficient of adhesion, the adhesive force transmitted by the vehicle wheels to the road can be reduced by reducing the gravity component of the vehicle wheels (by means of additional wheel units). It is evident from the measured test drive results. The waveforms of significant quantities describing the vehicle behaviour are comparable when comparing ASC with the sliding surface. A significant difference occurs in the body tilt angle of the experimental vehicle, where ASC, due to its design, limits the body tilt against the sliding surface. The maximum body tilt difference occurs when the ASC support wheels carry the minimum load. As the adhesive force transmitted by the vehicle wheels decreases, the difference in the ASC body angle with respect to the slip surface decreases. It is caused by a lower longitudinal deceleration value $-a_{x}$, which causes a lower fictitious force. Then the behaviour of the vehicle with ASC approximates the vehicle behaviour on the sliding surface. The current state of ASC is applicable for reducing the adhesive force, but needs to be further researched and optimized for more realistic body tilt.We see further research in mapping the dependence of the change in the adhesive force on the change in ASC stroke height. So that the size of the adhesive strength was similar slip surface.

The results from the experimental measurements are as follows:

- it is possible to reduce the adhesive force of the wheel by reducing the radial response on the vehicle wheel;

- using the ASC system, it is possible to change the adhesive force transmitted by the wheels of road vehicles;

- the measured parameters are mutually consistent with ASC and the sliding surface.

\section{References}

[1] Lee J. H. "Finite Element Modeling of Interfacial Forces and Contact Stresses of Pneumatic Tire on Fresh Snow for Combined Longitudinal and Lateral Slips". Journal of Terramechanics, vol. 48, č. 3, June2011, pp. 171-197.

[2] Lucet E. et al. "Dynamic Path Tracking Control of a Vehicle on Slippery Terrain". Control Engineering Practice, vol. 42, September 2015, pp. 60-73.

[3] Mavromatis S. et al. "Safety Assessment of Control Design Parameters through Vehicle Dynamics Model". Accident Analysis \& Prevention, vol. 125, April2019, pp. 330-335

[4] Kemzuraite K., et al. "Investigation of vehicle stability on road curves in winter conditions". Journal of KONES, vol. 18, No. 4, 2011, pp. 191-97.

[5] Šarkan, B., et al. "Vehicle coast-down method as a tool for calculating total resistance for the purposes of type-approval fuel consumption". ZeszytyNaukowe. Transport/PolitechnikaŚląska, vol. 98, 2018.

[6] Grip H. F. et al. "Nonlinear Vehicle Side-Slip Estimation with Friction Adaptation". Automatica, Vol. 44, No. 3, March2008, pp. 611-622.

[7] Jilek P., Voltr O., Pokorny J. "Simulation of adhesion conditions on road vehicles", Engineering mechanics 2016, Svratka, Czech republic, 2016, pp. 258-261.

[8] Jilek P., Šefčík I., Verner J., Berg J. "System allowing adhesion force change of road vehicle", 18th International Scientific Conference Engineering for Rural Development,Jelgava, Latvia, 2019, pp. 1876-1882. 
[9] Mavromatis S. et al. "Investigation of vehicle motion on sharp horizontal curves combined with steep longitudinal grades". Transportation Letters, vol. 8, No. 4, August2016, pp. 220-228.

[10] Novikov I., a Dmitriy L. "Experimental Installation for Calculation of Road Adhesion Coefficient of Locked Car Wheel”. Transportation Research Procedia, vol. 20, January2017, pp. 463-467.

[11] Pelc J. "Towards Realistic Simulation of Deformations and Stresses in Pneumatic Tyres". Applied Mathematical Modelling, vol. 31, No. 3, March2007, pp. 530-540. 